\title{
PRIMER REGISTRO DE UN PLATYRRHINI (ALOUATTINI) FÓSIL DEL CUATERNARIO DE COSTA RICA
}

\author{
César A. Laurito M. ${ }^{1}$ \& Ana Lucía Valerio Z. ${ }^{2}$ \\ ${ }^{1}$ Instituto Nacional de Aprendizaje, Núcleo de Turismo, \\ Apartado postal 203-2200, Coronado; San José - Costa Rica \\ E-mail: cesarlaurito@ice.co.cr \\ ${ }^{2}$ Departamento de Historia Natural, Museo Nacional de Costa Rica \\ Apartado Postal 749-1000, San José - Costa Rica \\ E-mail: alvalerio@costarricense.cr
}

(Recibido 13/9/04; Aceptado 15/3/05)

\begin{abstract}
This work describes some fossil remains, recovered from the Nacaome local fauna by the Archaeology Department of the Costa Rican National Museum during the campaign of 1993. The material includes a premolar tooth and a postcranial bone of a Platyrrhini monkey. The genus Alouatta is recognized by the anatomy of the third lower premolar which shares some affinities and differences with the living species of Alouatta palliata.

Keywords: Nicoya Peninsula, Costa Rica, Upper Pleistocene, Alouatta, Atelidae, Paleoecology

RESUMEN: Se describen algunos restos fósiles procedentes de la localidad de Nacaome, los cuales fueron recuperados por el departamento de antropología del Museo Nacional de Costa Rica durante la campaña de 1993. El material está compuesto por un premolar y un resto postcranial de un mono Platyrrhini. Se reconoció el género Alouatta a partir de la anatomía del tercer premolar inferior, el cual comparte algunas afinidades y diferencias con la especie viviente Aluatta palliata.

Palabras clave: Península de Nicoya, Costa Rica, Pleistoceno Superior, Alouatta, Atelidae, Paleoecología.
\end{abstract}

\section{INTRODUCCIÓN}

Los platirrinos fósiles son extremadamente raros, pero en los últimos años se ha incrementado el número de registros para el Pleistoceno y el Holoceno de las islas del Caribe, al menos siete especies endémicas distintivas son conocidas para las Grandes Antillas. Estos hallazgos incluyen tres especies para Jamaica, una para La Española y tres para Cuba (Rímoli, 1977; MacFadden, 1990, Ford, 1990; Rivero de la Calle \& Arredondo, 1991 y MacPhee et al. 1995). También se ha descrito un hallazgo para el Cuaternario de la isla de Granada (ver MacPhee et al., 2000). 
Fuera de las Antillas, en el Cenozoico de Colombia (Stirton, 1953 y Kay et al., 1989) son comunes los registros fósiles de monos neotropicales. A pesar de la proximidad geográfica de los hallazgos fósiles, América Central no contaba con ningún registro fósil hasta ahora.

El material procede de la Localidad de Nacaome, coordenadas 236.750 N y 391.500 W (Fig. 1) y fue recuperado por el Departamento de Antropología e Historia del Museo Nacional de Costa Rica en julio de 1992. Está constituido por una falange y un premolar, los cuales son descritos a continuación.
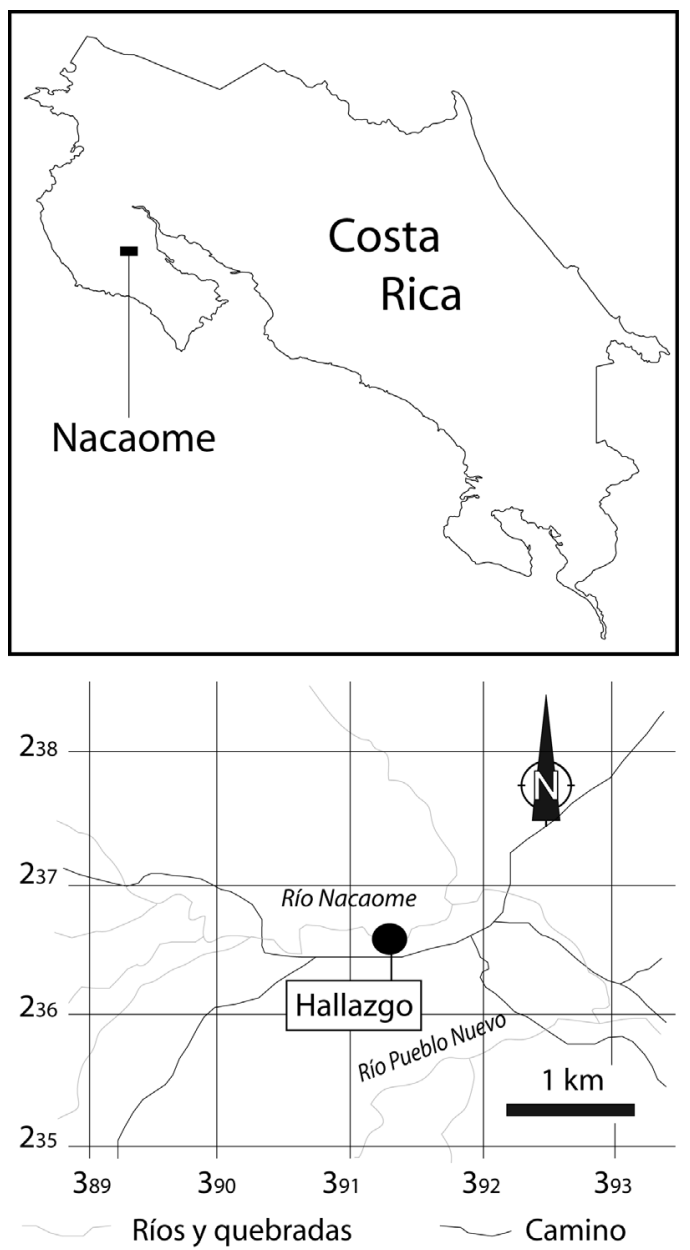

Fig. 1: Mapa de ubicación de la Localidad de Nacaome (tomado de la hoja topográfica Matambú, escala 1:50 000 IGNCR 3146 III)

\section{PALEONTOLOGÍA}

La sistemática utilizada está fundamentada en Rosenberger et al. (1990):

Infraorden PlatyrRHINI Geoffroy, 1812

Superfamilia ATEloIDEA Gray, 1825

Familia ATELIDAE Gray, 1825

Subfamilia AtelinAe Gray, 1825

Tribu Alouattini Trouessart, 1897

Género AlouATTA Lacépède, 1799

\section{Alouatta sp.}

Alouatta es uno de los primates más grandes del Neotrópico, mejor conocido como mono congo o mono aullador por las características vocalizaciones que emiten los machos adultos. Viven en tropas de hasta 11 individuos y están constituidos por varios machos y hembras adultos junto con infantes e individuos jóvenes. En Costa Rica habitan las tierras bajas hasta el bosque montano (2500 m.s.n.m.) y se distribuyen desde México hasta el Noroeste de América del Sur, se conocen alrededor de 12 especies de Alouatta (Glander, 1983).

Repositorio: el material que a continuación se describe se encuentra depositado en la colección paleontológica de la Sección de Geología del Museo Nacional bajo los códigos CFM-1397 y CFM-1398.

\section{DESCRIPCIÓN}

Tercer premolar inferior izquierdo: el ${ }_{3} \mathrm{Pm}$ CFM-1397, es de tipo caniniforme poco molariforme con corona grande y comprimida ligeramente en sentido bucolingual. El talónido es amplio pero de dimensiones ligeramente menores que el trigónido. El trigónido es más corto en sentido mesio distal y más ancho en sentido linguovestibular, presenta una única cúspide amplia y relativamente alta, convexa labialmente, con dos proyecciones laterales apenas perceptibles no observables en Alouatta palliata; el cíngulo labial está ausente, al igual que el cíngulo lingual; el talónido está rodeado de crestas poco notorias y no es posible diferenciarlas claramente a excepción de un hypoconulido poco conspicuo, el talónido es relativamente amplio si se le compara con los correspondientes talónidos de los terceros premolares inferiores de Alouatta palliata (Figs. 2a-d) y posee una notable depresión o cuenca en su área central. 




Fig. 2: Tercer premolar inferior izquierdo de Alouatta sp., pares estereográficos: a) vista oclusal, b) vista labial, c) vista distal y d) vista mesial. Falange tercera de la mano: e) vista lateral y f) vista ventral. Escala visual $=5 \mathrm{~mm}$. 
Falange tercera, bien preservada de $20 \mathrm{~mm}$ de longitud y de posición incierta, probablemente de la mano, código CFM- 1398 (Figs. 2e y f).

\section{DISCUSIÓN}

El ${ }_{3} \mathrm{Pm}$ que se describe, prácticamente no difiere en cuanto a su morfología de los correspondientes premolares de la actual Alouatta palliata; sin embargo, es posible establecer algunos caracteres que evidencian algún grado de diferenciación importante, como lo son: contorno de la corona oval más elongado y ligeramente más grande que en $A$. palliata; talónido más amplio con el hypoconulido mucho más reducido y raíz mucho más corta.

El material de estudio fue comparado con elementos dentales de un macho adulto de $A$. palliata, con desgaste avanzado de las superficies oclusales de los molares, a fin de asegurar que las comparaciones se hicieran con ejemplares de gran talla. Aún así el ejemplar de Nacaome supera ligeramente en talla la especie actual.

Se descartó el género Ateles, por cuanto su $\mathrm{Pm}_{3}$ se caracteriza por poseer un talónido con una área mucho más grande que su trigónido (cf. Tejedor, 2001).

\section{Paleoecología}

De acuerdo con las descripciones de la fauna Nacaome realizadas por Laurito et al. (1993) y el presente hallazgo de Alouatta sp., es posible asumir un escenario de sabanas arboladas donde pastaban y ramoneaban manadas de gliptodontes, caballos y mastodontes; con marcada estacionalidad (lo que fue deducido a partir de los anillos de crecimiento de la tortuga semiacuática Rhinoclemmys nicoyana, Acuña \& Laurito, 1996), con cursos fluviales y posible desarrollo de humedales estacionales. Debe tenerse presente que los sedimentos en los que se encontró el premolar son de origen fluvial.

\section{Edad}

La edad que se asume para este hallazgo es Pleistoceno Superior y se fundamenta en la megafauna asociada al mismo, a saber: Cuvieronius hyodon, Glyptotherium sp. y Equus sp. descrita por Laurito et al., 1993

\section{CONCLUSIONES}

Se describe el primer registro de un platirrino fósil para el Cuaternario de Costa Rica y América Central. Este pertenece a una especie desconocida de Alouatta que se asume es diferente de la actual A. palliata que habita el territorio nacional.

El registro de la localidad de Nacaome, es el más antiguo que se tiene hasta el momento de un Atelinae para América Central y constituye un importante elemento de la fauna Neotropical de abolengo suramericano en el Pleistoceno Superior de Costa Rica.

\section{REFERENCIAS}

ACUÑA, R.A. \& LAURITO, C.A., 1996: Nueva especie de Rhinoclemmys Fitzinger, 1836 (Chelonii, Cryptodira) del Cenozoico Tardío de Costa Rica. - Ameghiniana, Rev. Asoc. Paleontol. Argent. 33(3): 271-278.

FORD, S.F., 1990: Platyrrhine evolution in the West Indies. En: FLEAGLE, J.G \& ROSENBERGER, A.L. (eds.): The Platyrrhine Fossil Record. - J. Human Evolution, 19: 237-254.

GLANDER, K.E., 1983: Alouatta palliata (Congo, Howling Monkey, Howler Monkey). - En: JANZEN, D.H. (ed): Costa Rican natural history. - Univ. Chicago Press: 448-449.

KAY, R.F., MADDEN, R.H. \& GUERRERO DÍAZ, J., 1989: Nuevos hallazgos de monos en el Mioceno de Colombia. - Ameghiniana, Rev. Asoc. Paleontol. Argent. 25(3): 203-212.

LAURITO, C.A., VALERIO, W. \& VEGA, E., 1993: Nuevos hallazgos paleovertebradológicos en la península de Nicoya: implicancias paleoambientales y culturales de la fauna de Nacaome. - Rev. Geol. Amér. Central, 16: 113-115.

MacFADDEN, B.J., 1990: Chronology of Cenozoic primate localities in South America. - En: FLEAGLE, J.G \& ROSENBERGER, A.L. (eds.): The Platyrrhine Fossil Record. - J. Human Evolution, 19: 7-21. 
MacPHEE, R.D.E., SINGER, R. \& DIAMOND, M., 2000: Late Cenozoic land mammals from Grenada, Lesser Antilles Island-Arc. - Amer. Museum Novitates, 3302: 20 págs.

MacPHEE, R.D.E., HOROVITZ, I., ARREDONDO O. \& Jiménez Vásquez, O., 1995: A new genus for the extinct hispanolian monkey Saimiri bernensis Rímoli, 1977, with notes on its systematic position. - Amer. Museum Novitates, 3134: 21 págs.

RÍMOLI, R., 1977: Una nueva especie de mono (Cebidae: Saimirinae: Saimiri) de la Española. - Cuad. CENDIA, Univ. Aut. Santo Domingo, 242: 5-14.

RIVERO DE LA CALLE, M. \& ARREDONDO, O., 1991: Paralouatta varonai, a new Quaternary platyrrhine from Cuba. - J. Human Evolution, 21: 1-11.
ROSENBERGER, A., SETOGUCHI, T. \& SHIGEHARA, N., 1990: The fossil record of callitrichine primates. En: FLEAGLE, J.G \& ROSENBERGER, A.L. (eds.): The Platyrrhine Fossil Record. - J. Human Evolution, 19: 209-236.

STIRTON, R.A., 1953: Vertebrate paleontology and continental stratigraphy in Colombia. - Bull. Geol. Soc. Amer. 64: 603-622.

TEJEDOR, M.F., 2001: Aotus y los Atelinae: Nuevas evidencias en la sistemática de los primates platirrinos. Mastozoología Neotropical, 8(1): 41-57. 
